\title{
Agentes quelantes sulfidrílicos: uma revisão
}

Francielli Weber Santos

Cristina Wayne Nogueira

\section{Resumo}

O aumento do uso industrial de metais como o berílio, o cádmio, o cobre, o chumbo, o magnésio e o níquel inevitavelmente resultaram em um ambiente mais contaminado. Por esta razão, as intoxicações ambientais crônicas não são raras e resultam em uma preocupação para a saúde humana. A terapia que utiliza os agentes quelantes tem sido a base do tratamento médico de intoxicações por metal desde a década de 40 . Os agentes quelantes são utilizados clinicamente como antídotos para intoxicações agudas e crônicas por metal. Estes compostos atuam aumentando a excreção de elementos tóxicos, como o arsênio, o cádmio, o chumbo e o mercúrio os quais são bem conhecidos pela toxicidade que desenvolvem. Além disso, eles também diminuem a toxicidade do metal por prevenir a ligação destes á moléculas celulares alvo. Entretanto, existem muitas questões referentes à utilização terapêutica dos agentes quelantes ditiólicos, incluindo evidências de efeitos colaterais indesejáveis, ainda obscuras. Os aspectos expostos acima indicam claramente que experimentos mais detalhados e experimentos clínicos são necessários para melhor estabelecer as propriedades toxicológicas do BAL, DMPS e DMSA. 


\begin{abstract}
The increase industrial use of metals such as berilium, cadmium, copper, lead, magnesium and nickel results inevitably on a more contaminated environment. For this reason, environmental chronic poisonings with these metals have not been unusual and is a serious human health concern. Chelation therapy has been the basis for the medical treatment of metal poisoning for the past 4 decades. Chelating agents have been used clinically as antidotes for acute and chronic metal intoxications. These compounds enhance the excretion of toxic elements such as arsenic, cadmium, lead, or mercury, which are also well-known developmental toxicants. Moreover, they also decrease the metal's toxicity by preventing it from binding to cellular target molecules. However, there are many questions concerning the use of these compounds, including a wide range of overt and hidden undesirable side effects caused by these therapeutic dithiol chelating agents. The aspects pointed out above clearly indicate that more detailed experimental and clinical experiments are required to better establish the toxicological properties of BAL, DMPS and DMSA.
\end{abstract}

\title{
1. Histórico
}

\section{- 2,3-dimercaptopropanol ou dimercaprol (BAL)}

Os primeiros relatos do uso de agentes quelantes em casos de intoxicações datam da época da Segunda Guerra Mundial, na Inglaterra. Stocken e Thompson (48), em 1946, descreveram o uso do 2,3-dimercaptopropanol (BAL) como um antídoto para intoxicações pelo dicloro-vinil arsênio. Este composto é um potente agente tóxico presente em gases de guerra, conhecido como Lewisite, o qual é capaz de atuar nos pulmões, nos rins, outros órgãos internos ou outras superfícies do corpo. Segundo relatos, o BAL proporcionava $100 \%$ de sobrevivência em animais expostos topicamente ao Lewisite quando comparado a outros quelantes menos efetivos, como o monotiol 2-mercaptoetanol (48).

Após estes achados, Thompson testou os efeitos do BAL no seu próprio antebraço e relatou a eficácia deste composto para o uso em humanos. A partir destes estudos, o 2,3-dimercaptopropanol passou a ser conhecido como dimercaprol ou simplesmente BAL (British Anti-Lewisite) (48).

No final de 1947 intensificaram-se os estudos a respeito do BAL, especialmente na Inglaterra e nos EUA. Embora ele tenha 
sido desenvolvido como um antídoto para intoxicações por arsênio, este composto passou a ser indicado para o tratamento de intoxicações agudas por outros elementos tóxicos, como o mercúrio, o chumbo e o cádmio $(49,31,32,20)$.

\section{2.- Estrutura Química e Indicações Clínicas}

O BAL é um composto sulfidrílico, líquido, denso e incolor (6). Devido à sua lipossolubilidade e instabilidade em soluções aquosas, ele deve ser administrado dissolvido em óleo vegetal, através de injeção intramuscular (32).

A lipo ou hidrossolubilidade dos agentes quelantes é característica importante e pode limitar a sua utilização e eficácia. Compostos lipofílicos, como o BAL, podem facilmente atravessar a membrana celular e atingir os espaços intracelulares (2).

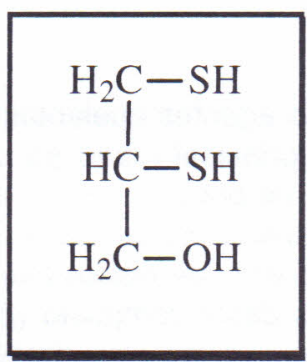

Fig. 1. Estrutura do 2,3- dimercaptopropanol (BAL)

A presença de dois grupos -SH vicinais é reconhecida como a estrutura essencial para a eficácia do agente quelante (38). Em intoxicações agudas por mercúrio, por exemplo, uma das terapêuticas fundamentais é utilizar compostos que apresentem na sua estrutura grupos sulfidrílicos (-SH), incluindo o BAL, a D-penicilamina $(36,47,32)$ e o ditiotreitol (DTT) (50). Estes compostos ditiólicos possuem a capacidade de complexar este metal pesado e aumentar a velocidade de excreção renal e biliar $(28,46)$.

Gilman e colaboradores (23), em 1946, propuseram que os metais pesados são tóxicos aos sistemas biológicos por formarem mercaptídeos reversíveis, a partir de grupos - $\mathrm{SH}$ de moléculas proteícas, tais como enzimas. 
Estes autores também postularam uma hipótese para explicar o mecanismo pelo qual os ditióis são capazes de reativar sistemas enzimáticos e exercer benefícios no tratamento de intoxicações com metais pesados. Segundo esta hipótese, o uso de quelantes ditiólicos proporcionaria a formação de mercaptídeos de baixa dissociabilidade, o que reverteria, efetivamente, a ligação dos metais pesados com sistemas proteicos sensíveis a estes (23).

As primeiras aplicações clínicas do BAL foram descritas para o tratamento de dermatites, decorrentes do uso terapêutico de compostos orgânicos contendo arsênio, usual na época para o tratamento de pacientes com sífilis (35). Outras indicações clínicas deste composto referem-se a sua utilização em formulações farmacêuticas. O Melarsoprolâ, uma preparação farmacêutica que contém BAL, é indicado no tratamento da doença do sono causada pelo Trypanosoma brucei gambiense. Este composto foi testado em camundongos infectados e demonstrou eficácia em eliminar os tripanossomas circulantes e inclusive do SNC $(26,43)$.

\section{Toxicidade}

A toxicidade dos agentes quelantes pode limitar o seu valor terapêutico. Esta é a principal razão para o declínio do uso do BAL e o aumento do uso de outros quelantes ditiólicos, tais como o DMPS (ácido 2,3-dimercapto-1-propanosulfônico) e o DMSA (ácido meso 2,3-dimercaptosuccínico), derivados mais hidrossolúveis e possivelmente menos tóxicos deste composto (2).

Dessa forma, devido às características estruturais e de solubilidade, a utilização do BAL apresenta várias desvantagens e, portanto tem sido questionada. Em torno de $50 \%$ dos pacientes tratados com este composto apresentam efeitos colaterais $(30,6)$, tais como, aumento da pressão sistólica e diastólica, taquicardia, naúseas, vômitos e dor de cabeça. Além disso, o BAL apresenta um baixo índice terapêutico, uma vez que a sua dose terapêutica efetiva é muito próxima da dose letal $(14,2)$. A DL50 para camundongos é $0,73 \mathrm{mmol} / \mathrm{kg}^{-1}$ (12). Deve ser injetado por via intramuscular, o que dificulta sua utilização (4).

Devido à sua lipossolubilidade, o BAL pode atravessar a membrana celular e atingir os espaços intracelulares (2), sendo completamente contra-indicado em intoxicações agudas por cloreto de cádmio (19). A formação do complexo metal-BAL aumenta a 
redistribuição tendo como conseqüência o acréscimo da deposição de cádmio no cérebro. A redistribuição de metais dos órgãos periféricos para o cérebro, após o tratamento com BAL, tem sido também descrito para outros metais, incluindo o arsênio, o metil mercúrio $(25,20)$, o mercúrio inorgânico (1) e o chumbo (17).

Como conseqüência, o BAL pode atingir níveis tóxicos em pacientes com nefropatia ou falência renal, situação comum em pacientes severamente intoxicados com cloreto de mercúrio, além de induzir sintomas clínicos tais como nervosismo, hiperatividade, hiperflexia e convulsões (51). Entretanto, em intoxicações agudas com arsênio o tratamento com BAL continua sendo fundamental (38).

Além disso, modelos experimentais mostraram que a administração de doses elevadas deste composto produz convulsões que culminam com a morte do animal, sugerindo ainda, que a modulação de receptores GABAérgicos, glutamatérgicos e canais iônicos estejam envolvidos no mecanismo de neurotoxicidade do BAL (39). Estudos do nosso grupo demonstraram ainda, que a toxicidade induzida pelo BAL pode estar relacionada com a inibição da captação e o aumento da liberação de glutamato em sinaptossomas de ratos (40), reafirmando o potencial neurotóxico do BAL.

Mesmo que diversos estudos tenham demonstrado que 0 BAL é capaz de induzir toxicidade, no Brasil, este agente quelante de metais tóxicos está na "Lista de medicamentos prioritários para registro de genéricos de 2002" da Agência Nacional de Vigilância Sanitária (ANVISA).

\section{Análogos Estruturais do $B A L$}

A estratégia terapêutica para tratar a intoxicação com metais envolve a administração crônica de agentes quelantes (13). Entretanto, um dos maiores problemas relacionados à eficiência dos agentes quelantes em intoxicações crônicas com metais pesados é a capacidade dos metais em induzir a síntese de proteínas de baixo peso molecular, metalotioneínas e a conseqüente ligação intracelular específica e de alta afinidade dos metais a estas proteínas.

Em 1990, Jones \& Cherian (27) descreveram as características ideais para que um agente quelante fosse efetivo no tratamento de intoxicações com metais pesados. Entre elas, a facilidade de entrar na célula, quelar os metais ligados a metalotioneínas e 
aumentar a excreção destes. Tudo isso, sem causar danos aos tecidos e sem aumentar os níveis do metal em outros órgãos críticos, tais como o cérebro.

Uma vez que a utilização do BAL apresenta diversas limitações e devido à sua toxicidade, outros agentes quelantes, potencialmente menos tóxicos, têm sido investigados (33).

Derivados estruturais do BAL, o DMPS (ácido 2,3dimercapto-1-propanosulfônico) e o DMSA (ácido meso 2,3dimercaptosuccínico) (Figuras 2 e 3, respectivamente), são compostos que apresentam dois grupos sulfidrílicos $(-\mathrm{SH})$ vicinais e caracterizam-se pela maior solubilidade em água e limitada solubilidade lipídica (3).

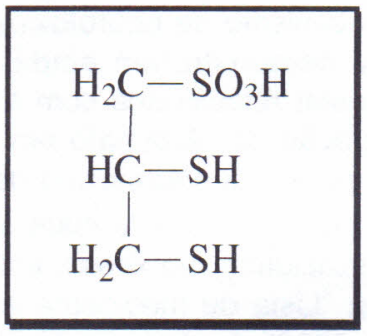

Fig. 2. Estrutura do ácido 2,3-dimercapto-1-propanosulfônico (DMPS)

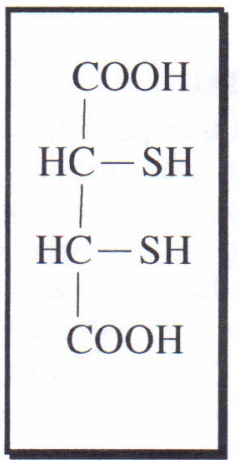

Fig. 3. Estrutura ácıdo meso '2,3- dimercaptosuccínico (DMSA) 
Várias são as vantagens do DMPS e do DMSA, em relação ao BAL. Apresentam-se como sólidos cristalinos e estáveis (6). São facilmente administrados por via oral (7). A DL50 em camundongos é $5.22 \mathrm{mmol} . \mathrm{kg}^{-1}$ para o DMPS e $16,5 \mathrm{mmol} . \mathrm{kg}^{-1}$ para o DMSA, portanto são maiores que a $\mathrm{DL}_{50}$ para o $\operatorname{BAL}\left(0,73 \mathrm{mmol} . \mathrm{kg}^{-1}\right)$. Devido a estas características, estes compostos são considerados menos tóxicos que o BAL (6).

O DMPS tem sido usado na antiga URSS desde 1958 e encontra-se disponível, comercialmente na Europa, como DIMAVAL ${ }^{\oplus}$. Além disso, este composto tem sido utilizado na Alemanha para o tratamento de intoxicações por mercúrio (11). Nos EUA, o DMSA (Succimer $^{\oplus}$ ) foi aprovado em 1990 pelo FDA (Food and Drug Admistration) apenas para o tratamento de intoxicações agudas e crônicas por chumbo em crianças com níveis sanguíneos deste metal maiores que $45 \mu \mathrm{g} / \mathrm{dl}$ (Department of Health and Human Services, 1991) (7). Sendo assim, o uso dessas drogas continua sendo experimental.

O DMPS foi descrito como uma droga efetiva no tratamento de intoxicações por mercúrio (34). Segundo diversos relatos, é uma alternativa segura e eficaz para substituir o $\operatorname{BAL}(51,11)$, apresentando menor toxicidade local e sistêmica e não causando redistribuição de mercúrio para o cérebro de ratos (7).

Entretanto, parece não ser efetivo no tratamento de intoxicações por chumbo e cádmio (27), embora não cause redistribuição de chumbo para o cérebro, em ratos (7).

A eficácia do DMSA foi originalmente descrita por pesquisadores chineses (53). É um quelante, oralmente efetivo, que pode ser usado no tratamento de crianças intoxicadas com chumbo, desde que apresentem niveis sangüíneos superiores a $45 \mathrm{mg} / \mathrm{dL}$ deste metal (41).

O tratamento terapêutico padrão recomendado para crianças com encefalopatia, induzida por chumbo, consta da associação de BAL e EDTA (16). Todavia, o DMSA parece ser efetivo em remover chumbo do cérebro (18). Portanto, quando comparado a outros quelantes, tais como BAL, EDTA e D-penicilamina, o DMSA é menos tóxico e é efetivo em reduzir a concentração sangüínea e aumentar a excreção urinária de chumbo em crianças (15) e animais. Outros estudos indicam que o DMSA pode ser mais efetivo que o BAL em proteger da nefrotoxicidade induzida por metil mercúrio (52).

Estudos clínicos estabeleceram que o DMSA é uma droga eficaz e segura e têm sugerido este composto como o quelante de 
preferência, em casos de intoxicações com metais (37). Contudo, o assunto apresenta algumas controvérsias; isto é, segundo alguns autores o DMSA apresenta a desvantagem de causar a redistribuição de chumbo dos orgãos periféricos para o cérebro $(18,22)$ e causar a eliminação de metais fisiologicamente essenciais. Porém, experiências recentes claramente relatam a distribuição extracelular do mesoDMSA e sua pouca eficiência em intoxicações com cádmio, visto que não retira o metal ligado a metalotioneínas (6).

Segundo Aposhian e colaboradores (6) (1992), o DMSA é três vezes menos tóxico que o DMPS. Entretanto, Aposhian \& Aposhian (5) (1990) sugerem precaução no uso do DMSA para gestantes, uma vez que apresenta efeitos embriotóxicos, em camundongos, quando administrado em altas doses.

O DMPS parece ser mais efetivo para remover cloreto de mercúrio em ratos, além de ser melhor agente terapêutico, pois mobiliza mercúrio mais rápido que o DMSA (10).

Recentemente, o DMPS e o DMSA têm sido utilizados para tratar intoxicações por mercúrio e chumbo e mostraram-se efetivos em estudos clínicos $(5,51)$.

Considerando o descrito acima, permanece em aberto a questão a respeito de qual seria a droga de escolha, em casos de intoxicação com metais pesados (38).

\section{Efeitos tóxicos dos quelantes sobre a enzima $\delta$-aminolevulinato desidratase}

Além dos possíveis efeitos tóxicos que os quelantes podem apresentar durante o tratamento de intoxicações por diversos metais tóxicos, sabe-se também que os agentes quelantes ditiólicos, em especial o BAL, apresentam atividade inibitória per se sobre a enzima $\delta$-aminolevulinato desidratase ( $\delta$-ALA-D) de tecido (20), assim como o DMPS e o DMSA (41).

Esta enzima catalisa uma importante reação que faz parte da rota biossintética dos compostos tetrapirrólicos (corrinas, bilinas, clorofilas e hemes). Os compostos tetrapirrólicos têm importância metabólica baseada, principalmente, na sua função como grupos prostéticos de proteínas.

A enzima d-ALA-D, devido a sua natureza sulfidrílica, pode ser inibida na presença de elementos como mercúrio $(44,20)$, chum- 
bo e outros que tenham alta afinidade por grupos sulfidrilicos. Esta enzima também é inibida por compostos orgânicos contendo telúrio e selênio $(8,21)$, os quais oxidam grupos sulfidrilicos.

Uma inibição da atividade da $\delta$-ALA-D, além de prejudicar a rota biossintética da molécula do heme pode resultar em um acúmulo do substrato ALA no fígado e no sangue, com conseqüente aumento na excreção urinária do mesmo (45).

Deficiências na via de biossíntese do heme são conhecidas como porfirias. A porfiria por deficiência da $\delta$-ALA-D, também conhecida por "porfiria aguda", pode ser adquirida, como ocorre em casos de intoxicação por chumbo (24), ou pode resultar de uma desordem recessiva autossômica, onde devido a uma quase completa ausência de atividade da enzima, os pacientes excretam altas quantidades de ALA urinário. Entretanto esta forma de porfiria é muito rara, havendo apenas quatro casos documentados no mundo (29).

O acúmulo de ALA também está relacionado com a superprodução de espécies reativas de oxigênio (9). Tem sido demonstrado que a oxidação do ALA, in vitro, pode provocar lipoperoxidação, lesões em mitocôndrias hepáticas e cerebrais, liberação de ferro da ferritina, lesões no DNA e inibição da adenilato ciclase cerebral (20). Os efeitos oxidativos do ALA podem estar associados ao aumento na atividade das enzimas antioxidantes superóxido dismutase e glutationa peroxidase observados em pacientes com porfiria aguda intermitente.

Recentemente, nosso grupo demonstrou que os quelantes ditiólicos, BAL, DMPS e DMSA, inibem a atividade da $\delta$-ALA-D de eritrócitos humanos, in vitro (41). Além disso, demonstrou-se também que os complexos formados pela interação do DMSA e DMPS com mercúrio ou cádmio teriam um maior poder inibitório sobre a atividade da $\delta$-ALA-D hepática do que os metais ou os quelantes isoladamente (42).

Tendo em vista os fatos acima mencionados, torna-se evidente a necessidade de maiores estudos nesta área, com o intuito de melhor entender os efeitos tóxicos e terapêuticos dos quelantes ditiólicos e, dessa forma auxiliar na escolha do agente mais eficaz para o tratamento de intoxicações por metais tóxicos. 


\section{Referências}

1.Aaseth, J., Jacobensem, D., Andersen, O \& Wickstrom, E. Treatment of mercury and lead poisoning with dimercaptosuccinic acid and sodium dimercaptopropanosulfate. Analyst. 120, 853-854 (1995).

2.Andersen, $\mathrm{O}$. Oral cadmium exposure in mice: Toxicokinetics and efficiency of chelating agents. Toxicol. 20 (2): 83-112 (1989).

3.Aposhian, H.V. DMSA and DMPS- Water soluble antidotes for heavy metal poisoning. Ann. Rev.Pharmacol. Toxicol. 23: 193-215 (1983).

4.Aposhian, H.V., Carter, D.E., Hoover, T.D., Hsu, C.A., Maiorino, R.M. \& Stine, E. DMSA, DMPS and DMPA as arsenic antidotes. Fundam.Appl.Toxicol. 4, S58-S70 (1984).

5.Aposhian, H.V \& Aposhian, M.M. Meso-2,3-dimercaptosuccinic acid: chemical, pharmacological and toxicological properties of na orally effective metal chelating agent. Ann. Rev. Pharmacol. Toxicol. 30, 279-306 (1990).

6.Aposhian, H.V., Maiorino, R.M., Rivera, M., Bruce, D.C., Dart, R.C., Hurlbut, K.M., Levine, D.J., Zheng, W., Quintus, F., Carter, D., Aposhian, M.M. Human studies with the chelating agents DMPS and DMSA. Clinical Toxicology. 30, 505-528 (1992).

7.Aposhian, M.M., Maiorino, R.M., Zhaofa, X. \& Aposhian, H.V. Sodium 2,3-dimercapto-1-propanesulfonate (DMPS) treatment does not redistribute lead or mercury to the brain of rats. Toxicology. 109, 4955 (1996).

8.Barbosa, N.B.V., Rocha, J.B.T., Zeni, G., Emanuelli, T., Beque, M.C., Braga, A.L. Effect of organic selenium on d-aminolevulinate dehydratase from liver, kidney, and brain of adult rats. Toxicol. Appl. Pharmacol. 149, 243-253 (1998).

9.Bechara, E.J.H., Medeiros, M.H.G., Monteiro, H.P., Hermes-Lima, M., Pereira, B., Demasi, M., Costa, C.A., Adballa, D.S.P., Onuki, J., Wendel, C.M. ${ }^{a}$, Masci, P.D. A free radical hypothesis of lead poisoning and inborn porphyrias associated with 5-aminolevulinic acid overload. Química Nova. 16, 385-392 (1993).

10.Buchet, J.P. \& Lauwerys, R.R. Influence of 2,3 dimercaptopropane1 -sulfonate and dimercaptosuccinic acid on the mobilization of mercury from tissues of rats pretreated with mercuric chloride, phenylmercury acetate or mercury vapors. Toxicology 54, 323-333 (1989).

11.Campbell, J.R., Clarkson, T.W. \& Omar, M.D. The terapeutic use of 2,3-dimercaptopropane-1-sulfonate in two cases of inorganic mercury poisoning. J. Am. Med. Assoc. 256, 3127-3130 (1986). 
12.Cantilena, L.R. \& Klaassen, C.D. Comparison of the effectiveness of several chelators after single administration on the toxicity, excretion and distribution of cadmium. Toxicol. Appl. Pharmacol. $58,452-460$ (1981).

13.Cantilena, L.R. \& Klaassen, C.D. The effect of chelating agents on the excretion of endogenous metals. Toxicol. Appl. Pharmacol. 63, 344-350 (1982).

14.Chisolm, J.J.Jr. Poisoning due to heavy metals. Pediat. Clin. North. Amer. 17, 591-615 (1970).

15.Chisolm, J.J.Jr. Evaluation of the potencial role of chelation therapy in treatment of low to moderate lead exposures. Environ. Health Perspect. 89, 67-74 (1990).

16.Chisolm, J.J.Jr. BAL, EDTA, DMSA and DMPS in the treatment of lead poisoning in children.Clin. Toxicol. 30(4), 493-504 (1992).

17.Cory-Slechta, D.A.; Weiss, B. \& Cox, C. Mobilization and redistribution of lead over the course of calcium disodium ethylenediamine tetraacetate chelation therapy. J. Pharmacol. Exp. Ther. 243, 804-813 (1987).

18.Cory-Slechta, D.A. Mobilization and redistribution of lead over the course of DMSA chelation therapy and long term efficacy. J.Pharmacol.Exp.Ther. 246, 84-91 (1988).

19.Dalhamn, T. \& Friberg, F. Dimercaprol (2,3 dimercaptopropanol) in chronic cadmium poisoning. Acta Pharmacol. 11, 68 (1955).

20.Emanuelli, T., Rocha, J.B.T., Pereira, M.E., Porciuncula, L.O, Morsch, V.M., Martins, AF. \& Souza, D.O. Effect of mercuric chloride intoxication and dimercaprol treatment on aminolevulinate dehydratase from brain, liver and kidney of adult mice. Pharmacol. \& Toxicol. 79, 138-143 (1996).

21.Farina, M., Folmer, V., Andrade, L.H., Zeni, G., Bolzan, R.C., Braga, A.L., Rocha, J.B.T. Selenoxides inhibit d-aminolevulinic acid dehydratase. Toxicol. Lett. 119, 27-37 (2001).

22.Flora, S.J.S.; Mathur, Seema \& Mathur, R. Effects of meso-2,3dimercaptosuccinic acid or 2,3-dimercaptopropane 1-sulfonate on beryllium-induced biochemical alterations and metal concentration in male rats. Toxicology 95, 167-175 (1995).

23.Gilman, A., Philips, F.S., Allen, R.P. and Koelle, E.S. The treatment of acute cadmium intoxication in rabbits with 2,3-dimercaptopropanol (BAL) and other mercaptans. Chemical Warfare Service 87, 85-101 (1946).

24.Granick, S. \& Mauzerall, D. Porphyrin biosynthesis in erythrocytes. 
II. Enzimes converting delta-aminolevulinic acid to coproporphyrinogen. J. Biol. Chem. 232, 1119-1140 (1958).

25. Hoover, T.D. \& Aposhian, H.V. BAL increases the arsenic-74 content of rabbit brain. Toxicol. Appl. Pharmacol. 70, 160-162 (1983).

26.Jennings, F.W., Atouguia, J.M. \& Murray, M. Topical chemotherapy for experimental murine African CNS-trypanosomiasis: The successful use of the arsenical, melarsoprol combined with the 5-nitroimidazole, fexinidazole or MK-436. Tropical Medicine \& International Health. 1, 590-598 (1996).

27.Jones, M.M. \& Cherian, M.G. The search for chelate antagonists for chronic cadmium intoxication. Toxicology 6, :1-25 (1990).

28. Jugo, S. The efficiency of chelating agents in eliminating $203 \mathrm{Hg}$ from the bodies of young and adult rats. Health Physics 38, 680682 (1980).

29.Kappas, A., Sassa, S., Galbraith, R.A., Nordmann, Y. The Phorphyrias. In: The Metabolic Bases of Inherited Disease, eds. Scriver, C.R., Beaudet, A.L., Sly, W.S., Valle, D. (McGraw Hill, New York), pp. 2103-2160 (1995).

30.Klaassen, C.D. Heavy metals and heavy-metal antagonists. In:A.G. Gilman, L.S. Goodman, T.W. Rall and F. Murad (Eds), The Pharmacological Basis of Therapeutics, Macmillan, New York, pp. 1605-1627 (1985).

31. Klaassen, C.D. Heavy metals and heavy-metals antagonists. In: The Pharmacological Basis of Therapeutics, eds Gilman, AG; Rall, T.W; Nies, AS. \& Taylor, P. 1592-1614. New York: Pergamon Press (1990).

32.Klaassen, C.D. Heavy metals and heavy-metals antagonists. In: The Pharmacological Basis of Therapeutics, Eds. Wonsiewicz, M.J. \& McCurdy,P. pp.1649-1671. New York: McGraw-Hill, (1996). 33.Keith, R.L.; Setiarahardjo, I.; Fernando, Q.; Aposhian, H.V. \& Gandolfi, A.J. Utilization of renal slices to evaluate the efficacy of chelating agents for removing mercury from the kidney. Toxicology 116, 67-75 (1997).

34.Kostygou, N.M. Pharmakol.Toksikol. 21, 64 (1958).

35.Longcope, W.T., Luetscher, J.A., Wintrobe, M.M. \& Juger, V. The treatment of arsenical dermatitis with preparations of BAL. J. Clin. Invest. 25, 528-533 (1946).

36.Mant, T.G.K.; Lewis, J.L.; Mattoo, T.K.; Ridgen, S.P.A.; Volans, G.N. \& House, I.M.; Wakefield, A.J. \& Cole, R.S. Mercury poisoning 
after disc-battery ingestion. Hum. Toxicol. 6 (2), 179-181 (1987).

37. Miller, A.L. Dimercaptosuccinic acid (DMSA), a non-toxic, watersoluble treatment for heavy metal toxicity. Altern. Med. Rev. 3(3), 199-207 (1998).

38.Muckter, H.; Liebl, B.; Reichl, F.X.; Hunder, G.; Walther, U. \& Fichtl, B. Are we ready to replace dimercaprol (BAL) as an arsenic antidote? Hum. Exp. Toxicol. 16(8), 460-465 (1997).

39.Nogueira, C.W., Soares, F.A, Bolzan, R.C., Jacques-Silva, M.C., Souza, D.O. and Rocha, J.B.T. Investigations into the mechanism of 2,3-dimercaptopropanol neurotoxicity. Neurochemical Research 25, 1553-1558 (2000).

40.Nogueira, C.W., Rotta, L.N., Tavares, R.G., Souza, D.O. and Rocha, J.B.T. BAL modulates glutamate transport in synaptosomes and synaptic vesicles from rat brain. NeuroReport 12, 511-514 (2001).

41.Nogueira, C.W., Soares, F.A., Nascimento, P.C., Muller, D., and Rocha, J.B.T. 2,3-dimercaptopropane-1-sulfonic acid and meso2,3- dimercaptosuccinic acid increase mercury and cadmiuminduced inhibition of d-aminolevulinate dehydratase. Toxicology 184, 85-95 (2003a).

42.Nogueira, C. W., Santos F.W., Soares, F.A., Rocha, J.B.T. 2,3Dimercaptopropanol, 2,3-Dimercaptopropane-1-Sulfonic Acid and meso-2, 3-Dimercaptosuccinic Acid Inhibit d-Aminolevulinate Dehydratase from Human Erythrocytes in vitro Environmental Research 2003b in press

43.Pappas, J.B.; Ahlquist, J.T.; Allen, E.M. \& Banner, W.Jr. Oral dimercaptosuccinic acid and ongoing exposure to lead: Effects on Heme synthesis and lead distribution in a rat model. Toxicol. Appl. Pharmacol. 133, 121-129 (1995).

44.Pepin, J., Milord, F., Khonde Na, Niyonsenga, T., Loko, L., Mpia, B. \& Dewals, P. Risk-factors for encephalopathy and mortality during melarsoprol treatment of Trypanosoma-Brucel-Gambiense sleeping sickness. Transactions of the Royal Society of Tropical Medicine and Hygiene. 89, 92-97(1995).

45.Rocha, J.B.T., Freitas, A.J., Marques, M.B., Pereira, M.E., Emanuelli, T., Souza, D.O. Effects of methylmercury exposure during the second stage of rapid postnatal brain growth on negative geotaxis and on delta-aminolevulinate dehydratase of suckling rats. Brazilian J. Med. Biol. Res. 26, 1077-1083 (1993).

46.Sassa, S., Fujita, H., Kappas, A. Genetic and chemical influences 
on heme biosynthesis. In: Kotyk, A., Skoda, J., Paces,V., Kostka, V. (Eds.), Highlights of Modern Biochemistry, Vol. 1. VSP, Utrecht, pp.329-338 (1989).

47.Shimada, H.; Fukudome, S.; Kiyozumi, M.; Funakoshi, T.; Adachi, T.; Yasutake, A. \& Kojima, S. Further study of effects of chelating agents on excretion of inorganic mercury in rats. Toxicology 77 , 157-169 (1993).

48.Singer, A.J.; Mofenson, H.C.; Caraccio, T.R. \& Ilasi, J. Mercury chloride poisoning due to ingestion of a stool fixative.J.Toxicol.Clin.Toxicol. 32(5), 577-582 (1994).

49.Stocken, L.A. \& Thompson, R.S.H. British anti-Lewisite 3. Arsenic and thiol excretion in animals after treatment of Lewisite burns. Biochem. J. 40, 548-554 (1946).

50.Stocken, L.A. British anti-Lewisite as an antidote to acute mercury poisoning. Biochem.J. 41, 358-360 (1947).

51.Suzuki, S.; Takamura, S.; Sugiki, K.; Imai, M.; Niwa, O. \& Tamatani, R. Protective effects of 2,3-dimercaptopropane-1sulfonate on mercury chloride-induced acute inhibition of enzymes from rat duodenal mucosa and kidney cortex. Toxicology $86,29-$ 48 (1994).

52.Toet, A.E.; Dijk, A.; Savelkoul, T.J.F. \& Meulenbelt, J. Mercury kinetics in a case of severe mercuric chloride poisoning treated with dimercapto-1-propane sulphonate (DMPS). Human \& Experimental Toxicology 13, 11-16 (1994).

53.Torre, A.; Belles, M.; Llobet, J.M.; Mayayo, E. \& Domingo, J.L. Comparasion of the effectiveness of 2,3-dimercaptopropanol and meso-2,3-dimercaptosuccinic acid (DMSA) as protective agents against mercuric chloride-induced nephrotoxicity in rats. Biol. Trace Elem. Res. 63(1), 1-10 (1998).

54.Wang, S.C.; Ting, K.S. \& Wu, C.C. Clin. Med. J. 84, 437 (1965).

Francielli Weber Santos

Cristina Wayne Nogueira

Departamento de Química - CCNE Universidade Federal de Santa Maria

Santa Maria, RS - Brasil 